IJMMS 2003:4, 229-240

PII. S0161171203201204

http://ijmms.hindawi.com

(c) Hindawi Publishing Corp.

\title{
CONTINUOUS DEPENDENCE OF SOLUTIONS IN MAGNETO-ELASTICITY THEORY
}

\author{
F. BOFILL and R. QUINTANILLA
}

Received 30 January 2002

\begin{abstract}
We prove continuous dependence on the intensity coefficient and continuous dependence on the external data in the theory of magneto-elasticity. We do not require the Lamé coefficients to be positive. We use logarithmic convexity arguments similar to those of Ames and Straughan (1992) in classical thermoelasticity.
\end{abstract}

2000 Mathematics Subject Classification: 74F15, 74H99.

1. Introduction. In recent years, much attention has been directed to the knowledge of existence, uniqueness, and continuous dependence in several thermomechanical situations. We recall the book of Ames and Straughan [2] where the energy method is widely considered as a tool to obtain qualitative properties of solutions. We focus our interest on coupling elastic effects with magnetic effects. A derivation of the equations and recent papers on magnetothermoelasticity and isothermal magneto-elasticity can be found in $[3,4,5,6$, $7,8,9,10,11,12]$.

In this paper, we consider the dynamical theory of magneto-elasticity. The system of equations is

$$
\begin{gathered}
\rho \mathbf{u}_{, t t}-\mu \Delta \mathbf{u}-(\lambda+\mu) \nabla \operatorname{div} \mathbf{u}-\alpha[\nabla \times \mathbf{h}] \times \mathbf{H}=\rho \mathbf{m}, \\
\beta \mathbf{h}_{, t}+\nabla \times[\nabla \times \mathbf{h}]-\beta \nabla \times[\mathbf{v} \times \mathbf{H}]=\rho \mathbf{r}, \\
\operatorname{div} \mathbf{h}=0,
\end{gathered}
$$

where $\mathbf{u}$ denotes the displacement, $\mathbf{v}=\mathbf{u}_{t}$ is the velocity, and $\mathbf{h}$ the magnetic field. A (known) constant magnetic field is denoted by $\mathbf{H}=(H, 0,0), \rho, \alpha$, and $\beta$ are positive constants, and $\mathbf{m}$ and $\mathbf{r}$ are the supply terms.

Here and from now on, we use summation and differentiation conventions: subscripts preceded by a comma denote partial differentiation with respect to the corresponding Cartesian coordinate; summation over repeated subscripts is implied.

The logarithmic convexity method is a very useful source of information about the qualitative behavior of the solutions of several kind of equations and systems (see, e.g., [2]). In particular, the method has been used to analyze the behavior of the solutions in classical thermoelasticity. Ames and Straughan [1] 
applied a logarithmic convexity technique to achieve continuous dependence on the supply terms and structural stability on the coupling term for the classical linear theory of thermoelasticity. They did not require the elasticity tensor to be sign-definite. All they needed was that the elasticity coefficients were symmetric.

The aim of this paper is to obtain a continuous dependence result on the intensity of the vector field $\mathbf{H}$ and the supply terms. Our main tool is also the logarithmic convexity method.

In this paper, we restrict our attention to homogeneous and isotropic materials. It is worth recalling that the extension to inhomogeneous and anisotropic materials would be straightforward.

Let $B$ be a bounded domain in the three-dimensional Euclidean space whose boundary $\partial B$ is smooth enough to allow the application of the divergence theorem. We assume that the set of equations (1.1), (1.2), and (1.3) holds in $B \times\left(0, t_{1}\right)$ for a time value $t_{1}<\infty$, and we assume the boundary conditions

$$
\mathbf{u}=0, \quad \mathbf{h} \cdot \mathbf{n}=0, \quad[\nabla \times \mathbf{h}] \times \mathbf{n}=0, \quad \text { on } \partial B \times(0, \infty),
$$

for all $t>0$. Here and from now on, we denote by $\mathbf{n}$ the normal vector to the boundary directed to the exterior. We impose the initial conditions

$$
\mathbf{u}(\mathbf{x}, 0)=\mathbf{f}(\mathbf{x}), \quad \mathbf{v}(\mathbf{x}, 0)=\mathbf{g}(\mathbf{x}), \quad \mathbf{h}(\mathbf{x}, 0)=\mathbf{h}_{0}(\mathbf{x}), \quad \text { in } B .
$$

For later use, we recall that the following inequality

$$
\int_{B}\left(h_{i} h_{i}+h_{i, j} h_{i, j}\right) d V \leq C \int_{B}\left(h_{i, j}-h_{j, i}\right)\left(h_{i, j}-h_{j, i}\right) d V
$$

holds with any vector field $\left(h_{i}\right)$ that satisfies (1.3) and the second and third equalities of (1.4). Here, $C$ is a constant that depends on the domain $B$.

Here are the contents of the paper. In Section 2, we prove some lemmas and we state some other preliminaries. In Section 3, we prove the continuous dependence result.

2. Preliminaries. We denote by $\left(u_{i}^{(1)}, h_{i}^{(1)}\right)$ the solution corresponding to the external data $\left(m_{i}^{(1)}, r_{i}^{(1)}\right)$ and intensity $H^{(1)}$. Let $\left(u_{i}^{(2)}, h_{i}^{(2)}\right)$ be the solution corresponding to the external data $\left(m_{i}^{(2)}, r_{i}^{(2)}\right)$ and intensity $H^{(2)}$. We introduce the notation

$$
\begin{gathered}
w_{i}=u_{i}^{(2)}-u_{i}^{(1)}, \quad l_{i}=h_{i}^{(2)}-h_{i}^{(1)}, \quad K=H^{(2)}-H^{(1)}, \quad \mathbf{K}=(K, 0,0), \\
F_{i}=\rho\left(m_{i}^{(2)}-m_{i}^{(1)}\right), \quad R_{i}=\rho\left(r_{i}^{(2)}-r_{i}^{(1)}\right) .
\end{gathered}
$$


It follows that $\left(w_{i}, l_{i}\right)$ satisfies the system

$$
\begin{gathered}
\rho \mathbf{w}_{, t t}-\mu \Delta \mathbf{w}-(\lambda+\mu) \nabla \operatorname{div} \mathbf{w}-\alpha[\nabla \times \mathbf{l}] \times \mathbf{H}^{(1)}-\alpha\left[\nabla \times \mathbf{h}^{(2)}\right] \times \mathbf{K}=\mathbf{F}, \\
\beta \mathbf{l}_{, t}+\nabla \times[\nabla \times \mathbf{l}]-\beta \nabla \times\left[\dot{\mathbf{w}} \times \mathbf{H}^{(1)}\right]-\beta \nabla \times\left[\mathbf{v}^{(2)} \times \mathbf{K}\right]=\mathbf{R}, \\
\operatorname{div} \mathbf{l}=0,
\end{gathered}
$$

the boundary conditions

$$
\mathbf{w}=0, \quad \mathbf{l} \cdot \mathbf{n}=0, \quad[\nabla \times \mathbf{l}] \times \mathbf{n}=0, \quad \text { on } \partial B \times(0, \infty),
$$

and the initial conditions

$$
\mathbf{W}(\mathbf{x}, 0)=\dot{\mathbf{w}}(0, \mathbf{x})=\mathbf{l}(\mathbf{x}, 0)=0, \quad \text { in } B .
$$

LEMMA 2.1. Let

$$
V(t)=\int_{0}^{t} \int_{B}\left[\rho \dot{\mathbf{w}} \dot{\mathbf{w}}+\mu \nabla \mathbf{w} \cdot \nabla \mathbf{w}+(\lambda+\mu)(\operatorname{div} \mathbf{w})^{2}+\alpha \mathbf{l} \cdot \mathbf{l}\right] d V d s .
$$

Then,

$$
\begin{aligned}
V(t)=2 \int_{0}^{t} \int_{B}(t-s)[ & F_{i} \dot{w}_{i}+S_{i} l_{i}+\alpha K\left(\dot{w}_{2}\left(h_{2,1}^{(2)}-h_{1,2}^{(2)}\right)+\dot{w}_{3}\left(h_{3,1}^{(2)}-h_{1,3}^{(2)}\right)\right) \\
& +K \alpha\left(l_{1}\left(-\dot{u}_{2,2}^{(2)}-\dot{u}_{3,3}^{(2)}\right)+l_{2} \dot{u}_{2,1}^{(2)}+l_{3} \dot{u}_{3,1}^{(2)}\right) \\
& \left.-\frac{\alpha}{\beta}\left(l_{i, j}-l_{j, i}\right)\left(l_{i, j}-l_{j, i}\right)\right] d V d s
\end{aligned}
$$

where

$$
S_{i}(t)=\frac{\alpha}{\beta} R_{i}(t)
$$

Proof. Differentiate twice and use the evolution equations and the boundary conditions to obtain

$$
\begin{aligned}
\frac{d^{2} V}{d t^{2}}= & 2 \int_{B}\left[\mu w_{i, j} \dot{w}_{i, j}+(\lambda+\mu) w_{i, i} \dot{w}_{j, j}+\rho \dot{w}_{i} \ddot{w}_{i}+\alpha l_{i} \dot{l}_{i}\right] d V \\
= & 2 \int_{B}\left[\mu w_{i, j} \dot{w}_{i, j}+(\lambda+\mu) w_{i, i} \dot{w}_{j, j}\right] d V \\
& -2 \int_{B}\left[\mu w_{i, j} \dot{w}_{i, j}+(\lambda+\mu) w_{i, i} \dot{w}_{j, j}-\alpha H^{1}\left(\dot{w}_{2}\left(l_{2,1}-l_{1,2}\right)+\dot{w}_{3}\left(l_{3,1}-l_{1,3}\right)\right)\right] d V \\
& +2 \int_{B}\left[F_{i} \dot{w}_{i}+\alpha K\left(\dot{w}_{2}\left(h_{2,1}^{(2)}-h_{1,2}^{(2)}\right)+\dot{w}_{3}\left(h_{3,1}^{(2)}-h_{1,3}^{(2)}\right)\right)\right] d V \\
& -2 \int_{B}\left[\alpha H^{1}\left(\dot{w}_{2}\left(l_{2,1}-l_{1,2}\right)+\dot{w}_{3}\left(l_{3,1}-l_{1,3}\right)\right)\right] d V
\end{aligned}
$$




$$
\begin{aligned}
&+2 \int_{B}[ S_{i} l_{i}+K \alpha\left(l_{1}\left(-\dot{u}_{2,2}^{(2)}-\dot{u}_{3,3}^{(2)}\right)+l_{2} \dot{u}_{2,1}^{(2)}+l_{3} \dot{u}_{3,1}^{(2)}\right) \\
&\left.-\frac{\alpha}{\beta}\left(l_{i, j}-l_{j, i}\right)\left(l_{i, j}-l_{j, i}\right)\right] d V \\
&=2 \int_{B}\left[F_{i} \dot{w}_{i}+S_{i} l_{i}+\alpha K\left(\dot{w}_{2}\left(h_{2,1}^{(2)}-h_{1,2}^{(2)}\right)+\dot{w}_{3}\left(h_{3,1}^{(2)}-h_{1,3}^{(2)}\right)\right)\right. \\
&\left.\quad+K \alpha\left(l_{1}\left(-\dot{u}_{2,2}^{(2)}-\dot{u}_{3,3}^{(2)}\right)+l_{2} \dot{u}_{2,1}^{(2)}+l_{3} \dot{u}_{3,1}^{(2)}\right)-\frac{\alpha}{\beta}\left(l_{i, j}-l_{j, i}\right)\left(l_{i, j}-l_{j, i}\right)\right] d V .
\end{aligned}
$$

The lemma follows after two quadratures and from the equality

$$
\int_{0}^{t}\left(\int_{0}^{s} f(\tau) d \tau\right) d s=\int_{0}^{t}(t-s) f(s) d s
$$

which is satisfied by every function $f(s)$.

It will be useful to introduce the notation

$$
P_{i}(t)=\int_{0}^{t} l_{i}(s) d s
$$

To obtain our results, we define the function

$$
H(t)=\int_{0}^{t} \int_{B}\left(\rho w_{i} w_{i}+\frac{\alpha}{\beta}(t-s)\left(P_{i, j}-P_{j, i}\right)\left(P_{i, j}-P_{j, i}\right)\right) d V d s .
$$

It is clear that

$$
\frac{d H}{d t}=2 \int_{0}^{t} \int_{B}\left(\rho w_{i} \dot{w}_{i}+\frac{\alpha}{2 \beta}\left(P_{i, j}-P_{j, i}\right)\left(P_{i, j}-P_{j, i}\right)\right) d V d s .
$$

The second derivative of the function $H$ is given in the next lemma.

LEMMA 2.2. The second derivative of the function $H$ is

$$
\begin{aligned}
\frac{d^{2} H}{d t^{2}}= & 4 \int_{0}^{t} \int_{B}\left[\rho \dot{w}_{i} \dot{w}_{i}+\frac{\alpha}{\beta}(t-s)\left(l_{i, j}-l_{j, i}\right)\left(l_{i, j}-l_{j, i}\right)\right] d V d s \\
- & -4 \int_{0}^{t} \int_{B}(t-s)\left[\left(F_{i} \dot{w}_{i}+S_{i} l_{i}\right)+\alpha K\left(\dot{w}_{2}\left(h_{2,1}^{(2)}-h_{1,2}^{(2)}\right)+\dot{w}_{3}\left(h_{3,1}^{(2)}-h_{1,3}^{(2)}\right)\right)\right. \\
& \left.+K \alpha\left(l_{1}\left(-\dot{u}_{2,2}^{(2)}-\dot{u}_{3,3}^{(2)}\right)+l_{2} \dot{u}_{2,1}^{(2)}+l_{3} \dot{u}_{3,1}^{(2)}\right)\right] d V d s \\
+ & 2 \int_{B}\left[\left(F_{i} w_{i}+Q_{i} l_{i}\right)+\alpha K\left(w_{2}\left(h_{2,1}^{(2)}-h_{1,2}^{(2)}\right)+w_{3}\left(h_{3,1}^{(2)}-h_{1,3}^{(2)}\right)\right)\right] d V \\
+ & 2 \int_{B}\left[K \alpha\left(l_{1}\left(-u_{2,2}^{(2)}-u_{3,3}^{(2)}\right)+l_{2} u_{2,1}^{(2)}+l_{3} u_{3,1}^{(2)}\right)\right. \\
& \left.-K \alpha\left(l_{1}\left(-f_{3,3}-f_{2,2}\right)+l_{2} f_{2,1}+l_{3} f_{3,1}\right)\right] d V
\end{aligned}
$$


where

$$
Q_{i}(t)=\int_{0}^{t} S_{i}(s) d s
$$

Proof. A direct differentiation gives

$$
\frac{d^{2} H}{d t^{2}}=2 \int_{0}^{t} \int_{B} \rho \dot{w}_{i} \dot{w}_{i} d V d s+2 \int_{0}^{t} \int_{B}\left[\rho w_{i} \ddot{w}_{i}+\frac{\alpha}{\beta}\left(P_{i, j}-P_{j, i}\right)\left(l_{i, j}-l_{j, i}\right)\right] d V d s .
$$

Now, we make some calculations to determine the evolution of the second integral. If we multiply (2.2) by $w_{i}$, and integrate over $B$, we obtain

$$
\begin{aligned}
\int_{B} \rho w_{i} \ddot{w}_{i} d V \\
=-\int_{B}\left[\mu w_{i, j} w_{i, j}+(\lambda+\mu) w_{i, i} w_{j, j}-\alpha H^{1}\left(w_{2}\left(l_{2,1}-l_{1,2}\right)+w_{3}\left(l_{3,1}-l_{1,3}\right)\right)\right] d V \\
\quad+\int_{B}\left[F_{i} w_{i}+\alpha K\left(w_{2}\left(h_{2,1}^{(2)}-h_{1,2}^{(2)}\right)+w_{3}\left(h_{3,1}^{(2)}-h_{1,3}^{(2)}\right)\right)\right] d V .
\end{aligned}
$$

If we integrate (2.3) with respect to the time parameter, multiply it by $l_{i}$, and integrate over $B$, we obtain

$$
\begin{aligned}
& \frac{\alpha}{\beta} \int_{B} l_{i} l_{i} d V \\
&=-\int_{B}\left[\alpha H^{1}\left(w_{2}\left(l_{2,1}-l_{1,2}\right)+w_{3}\left(l_{3,1}-l_{1,3}\right)\right)-\frac{\alpha}{\beta}\left(P_{i, j}-P_{j, i}\right)\left(l_{i, j}-l_{j, i}\right)\right] d V \\
&+\int_{B}\left[Q_{i} l_{i}+K \alpha\left(l_{1}\left(-u_{2,2}^{(2)}-u_{3,3}^{(2)}\right)+l_{2} u_{2,1}^{(2)}+l_{3} u_{3,1}^{(2)}\right)\right. \\
&\left.\quad-K \alpha\left(l_{1}\left(-f_{3,3}-f_{2,2}\right)+l_{2} f_{2,1}+l_{3} f_{3,1}\right)\right] d V .
\end{aligned}
$$

It follows that

$$
\begin{aligned}
& \int_{B}\left(\rho w_{i} \ddot{w}_{i}+\frac{\alpha}{\beta}\left(P_{i, j}-P_{j, i}\right)\left(l_{i, j}-l_{j, i}\right)\right) d V \\
& =-\int_{B}\left[\mu w_{i, j} w_{i, j}+(\lambda+\mu) w_{i, i} w_{j, j}+\frac{\alpha}{\beta} l_{i} l_{i}\right] d V \\
& +\int_{B}\left[\left(F_{i} w_{i}+Q_{i} l_{i}\right)+\alpha K\left(w_{2}\left(h_{2,1}^{(2)}-h_{1,2}^{(2)}\right)+w_{3}\left(h_{3,1}^{(2)}-h_{1,3}^{(2)}\right)\right)\right] d V \\
& +\int_{B}\left[K \alpha\left(l_{1}\left(-u_{2,2}^{(2)}-u_{3,3}^{(2)}\right)+l_{2} u_{2,1}^{(2)}+l_{3} u_{3,1}^{(2)}\right)\right. \\
& \left.\quad-K \alpha\left(l_{1}\left(-f_{3,3}-f_{2,2}\right)+l_{2} f_{2,1}+l_{3} f_{3,1}\right)\right] d V
\end{aligned}
$$


Then, we obtain

$$
\begin{aligned}
\frac{d^{2} H}{d t^{2}}= & 4 \int_{0}^{t} \int_{B} \rho \dot{w}_{i} \dot{w}_{i} d V d s \\
& -2 \int_{0}^{t} \int_{B}\left[\mu w_{i, j} w_{i, j}+(\lambda+\mu) w_{i, i} w_{j, j}+\frac{\alpha}{\beta} l_{i} l_{i}+\rho \dot{w}_{i} \dot{w}_{i}\right] d V d s \\
& +2 \int_{B}\left[\left(F_{i} w_{i}+Q_{i} l_{i}\right)+\alpha K\left(w_{2}\left(h_{2,1}^{(2)}-h_{1,2}^{(2)}\right)+w_{3}\left(h_{3,1}^{(2)}-h_{1,3}^{(2)}\right)\right)\right] d V \\
+ & 2 \int_{B}\left[K \alpha\left(l_{1}\left(-u_{2,2}^{(2)}-u_{3,3}^{(2)}\right)+l_{2} u_{2,1}^{(2)}+l_{3} u_{3,1}^{(2)}\right)\right. \\
& \left.-K \alpha\left(l_{1}\left(-f_{3,3}-f_{2,2}\right)+l_{2} f_{2,1}+l_{3} f_{3,1}\right)\right] d V
\end{aligned}
$$

Lemma 2.2 is a consequence of Lemma 2.1 and equality (2.21).

We now state a lemma concerning the behaviour of the magnetic field, which will also be used in the next section.

LEMMA 2.3. There exist three positive constants $A, B^{*}$, and $C^{*}$ such that

$$
\int_{0}^{t} \int_{B} l_{i} l_{i} d V d s \leq \int_{0}^{t_{1}} \int_{B}\left[A S_{i} S_{i}+B^{*} \rho \dot{w}_{i} \dot{w}_{i}+C^{*} K^{2}\right] d V d s
$$

for $t \leq t_{1}$.

Proof. In view of (2.3), we have

$$
\begin{aligned}
\int_{0}^{t} \int_{B} l_{i} l_{i} d V d s= & \frac{1}{\beta} \int_{0}^{t} \int_{B} \beta l_{i} l_{i} d V d s \\
= & -\frac{1}{\beta} \int_{0}^{t} \int_{B} \frac{\partial}{\partial s}\left[(t-s) \beta l_{i} l_{i}\right] d V d s \\
& +\frac{2}{\beta} \int_{0}^{t} \int_{B}(t-s) \beta l_{i} \frac{\partial l_{i}}{\partial s} d V d s \\
= & \frac{2}{\beta} \int_{0}^{t} \int_{B}(t-s)\left[R_{i} l_{i}-\left(l_{i, j}-l_{j, i}\right)\left(l_{i, j}-l_{j, i}\right)\right. \\
& -\beta H^{(1)}\left(\dot{w}_{2}\left(l_{2,1}-l_{1,2}\right)+\dot{w}_{3}\left(l_{3,1}-l_{1,3}\right)\right) \\
& \left.-\beta K\left(\dot{u}_{2}^{(2)}\left(l_{2,1}-l_{1,2}\right)+\dot{u}_{3}^{(2)}\left(l_{3,1}-l_{1,3}\right)\right)\right] d V d s .
\end{aligned}
$$


The use of the arithmetic-geometric mean inequality leads to the following estimates:

$$
\begin{aligned}
& \int_{0}^{t} \int_{B}(t-s) R_{i} l_{i} d V d s \leq \frac{\epsilon_{1}}{2} \int_{0}^{t} \int_{B} t_{1} R_{i} R_{i} d V d s+\frac{1}{2 \epsilon_{1}} \int_{0}^{t} \int_{B} t_{1} l_{i} l_{i} d V d s \\
& \int_{0}^{t} \int_{B}(t-s)\left(\dot{w}_{2}\left(l_{2,1}-l_{1,2}\right)+\dot{w}_{3}\left(l_{3,1}-l_{1,3}\right)\right) d V d s \\
& \leq \frac{\epsilon_{2}}{2} \int_{0}^{t} \int_{B} t_{1}\left(l_{i, j}-l_{j, i}\right)\left(l_{i, j}-l_{j, i}\right) d V d s+\frac{1}{2 \epsilon_{2}} \int_{0}^{t} \int_{B} t_{1} \dot{w}_{i} \dot{w}_{i} d V d s \\
& \int_{0}^{t} \int_{B}(t-s) K\left(\dot{u}_{2}^{(2)}\left(l_{2,1}-l_{1,2}\right)+\dot{u}_{3}^{(2)}\left(l_{3,1}-l_{1,3}\right)\right) d V d s \\
& \leq \frac{\epsilon_{3}}{2} \int_{0}^{t} \int_{B} t_{1} K^{2} d V d s+\frac{1}{2 \epsilon_{3}} \int_{0}^{t} \int_{B} t_{1} \dot{u}_{i}^{(2)}\left(l_{i, j}-l_{j, i}\right)\left(l_{i, j}-l_{j, i}\right) d V d s,
\end{aligned}
$$

where $\epsilon_{1}, \epsilon_{2}$, and $\epsilon_{3}$ are arbitrary positive constants.

If we assume that $\dot{u}_{i}^{(2)}$ is uniformly bounded on the interval $\left[0, t_{1}\right]$, we can make a suitable choice of the parameters $\epsilon_{i}(i=1,2,3)$ to obtain the estimate (2.22), where $A, B^{*}$, and $C^{*}$ can be easily computed.

3. Continuous dependence. In this section, we obtain continuous dependence and structural stability results. We assume that the functions

$$
\sup _{B}\left|h_{i, j}^{(2)}\right|^{2}, \quad \sup _{B}\left|\dot{u}_{i, j}^{(2)}\right|^{2}, \quad \sup _{B}\left|u_{i, j}^{(2)}-f_{i, j}\right|^{2},
$$

are uniformly bounded by a constant $M$.

Here, we introduce a family of functions

$$
H_{\omega}(t)=H(t)+\omega
$$

where $\omega$ is an arbitrary positive constant.

LEMMA 3.1. Let

$$
\omega=\int_{0}^{t_{1}} \int_{B}\left(F_{i} F_{i}+2 K^{2}+S_{i} S_{i}+Q_{i} Q_{i}\right) d V d s .
$$

Then, there exists a positive constant $\xi$ such that

$$
H_{\omega} \frac{d^{2} H_{\omega}}{d t^{2}}-\left(\frac{d H_{\omega}}{d t}\right)^{2} \geq-\xi H_{\omega}^{2}
$$

for $t \leq t_{1}$. 
Proof. From the definition of $H_{\omega}$ and Lemma 2.2, it follows that

$$
\begin{aligned}
& H_{\omega} \frac{d^{2} H_{\omega}}{d t^{2}}-\left(\frac{d H_{\omega}}{d t}\right)^{2} \\
& =4 N^{2}+4 \omega \int_{0}^{t} \int_{B}\left(\rho \dot{w}_{i} \dot{w}_{i}+(t-s) \frac{\alpha}{\beta}\left(l_{i, j}-l_{j, i}\right)\left(l_{i, j}-l_{j, i}\right)\right) d V d s \\
& -H_{\omega}\left(4 \int _ { 0 } ^ { t } \int _ { B } ( t - s ) \left[\left(F_{i} \dot{w}_{i}+S_{i} l_{i}\right)+\alpha K\left(\dot{w}_{2}\left(h_{2,1}^{(2)}-h_{1,2}^{(2)}\right)+\dot{w}_{3}\left(h_{3,1}^{(2)}-h_{1,3}^{(2)}\right)\right)\right.\right. \\
& \left.\quad+K \alpha\left(l_{1}\left(-\dot{u}_{2,2}^{(2)}-\dot{u}_{3,3}^{(2)}\right)+l_{2} \dot{u}_{2,1}^{(2)}+l_{3} \dot{u}_{3,1}^{(2)}\right)\right] d V d s \\
& +2 \int_{0}^{t} \int_{B}\left[\left(F_{i} w_{i}+Q_{i} l_{i}\right)+\alpha K\left(w_{2}\left(h_{2,1}^{(2)}-h_{1,2}^{(2)}\right)+w_{3}\left(h_{3,1}^{(2)}-h_{1,3}^{(2)}\right)\right)\right] d V \\
& +2 \int_{B}\left[K \alpha\left(l_{1}\left(-u_{2,2}^{(2)}-u_{3,3}^{(2)}\right)+l_{2} u_{2,1}^{(2)}+l_{3} u_{3,1}^{(2)}\right)\right. \\
& \left.\left.\quad-K \alpha\left(l_{1}\left(-f_{3,3}-f_{2,2}\right)+l_{2} f_{2,1}+l_{3} f_{3,1}\right)\right] d V d s\right),
\end{aligned}
$$

where

$$
\begin{aligned}
N^{2} & =I_{1} I_{2}-I_{3}^{2}, \\
I_{1} & =\int_{0}^{t} \int_{B}\left(\rho w_{i} w_{i}+(t-s) \frac{\alpha}{\beta}\left(P_{i, j}-P_{j, i}\right)\left(P_{i, j}-P_{j, i}\right)\right) d V d s, \\
I_{2} & =\int_{0}^{t} \int_{B}\left[\rho \dot{w}_{i} \dot{w}_{i}+(t-s) \frac{\alpha}{\beta}\left(l_{i, j}-l_{j, i}\right)\left(l_{i, j}-l_{j, i}\right)\right] d V d s, \\
I_{3} & =\int_{0}^{t} \int_{B}\left(\rho w_{i} \dot{w}_{i}+(t-s) \frac{\alpha}{\beta}\left(P_{i, j}-P_{j, i}\right)\left(l_{i, j}-l_{j, i}\right)\right) d V d s .
\end{aligned}
$$

In view of the Schwarz inequality, we have $N^{2} \geq 0$.

Now, we estimate some integrals which are on the right-hand side of equality (3.5). After some uses of the Hölder inequality and inequality (1.6), we can obtain the existence of constants $a_{i}$ such that

$$
\begin{aligned}
& \int_{0}^{t} \int_{B}(t-s) F_{i} \dot{w}_{i} d V d s \leq a_{1}\left(\int_{0}^{t} \int_{B} \rho \dot{w}_{i} \dot{w}_{i} d V d s\right)^{1 / 2}\left(\int_{0}^{t} \int_{B} F_{i} F_{i} d V d s\right)^{1 / 2}, \\
& \int_{0}^{t} \int_{B}(t-s) S_{i} l_{i} d V d s \leq a_{2}\left(\int_{0}^{t} \int_{B}(t-s)\left(l_{i, j}-l_{j, i}\right)\left(l_{i, j}-l_{j, i}\right) d V d s\right)^{1 / 2} \\
& \times\left(\int_{0}^{t} \int_{B} S_{i} S_{i} d V d s\right)^{1 / 2} \\
& \int_{0}^{t} \int_{B}(t-s) \alpha K\left(\dot{w}_{2}\left(h_{2,1}^{(2)}-h_{1,2}^{(2)}\right)+\dot{w}_{3}\left(h_{3,1}^{(2)}-h_{1,3}^{(2)}\right)\right) d V d s \\
& \leq a_{3}\left(\int_{0}^{t} \int_{B} \rho \dot{w}_{i} \dot{w}_{i} d V d s\right)^{1 / 2}\left(\int_{0}^{t} \int_{B} K^{2} d V d s\right)^{1 / 2},
\end{aligned}
$$




$$
\begin{aligned}
& \int_{0}^{t} \int_{B}(t-s) K \alpha\left(l_{1}\left(-\dot{u}_{2,2}^{(2)}-\dot{u}_{3,3}^{(2)}\right)+l_{2} \dot{u}_{2,1}^{(2)}+l_{3} \dot{u}_{3,1}^{(2)}\right) d V d s \\
& \leq a_{4}\left(\int_{0}^{t} \int_{B}(t-s)\left(l_{i, j}-l_{j, i}\right)\left(l_{i, j}-l_{j, i}\right) d V d s\right)^{1 / 2} \\
& \quad \times\left(\int_{0}^{t} \int_{B} K^{2} d V d s\right)^{1 / 2} .
\end{aligned}
$$

From (3.7), it follows that

$$
\begin{aligned}
& \int_{0}^{t} \int_{B}(t-s)\left[\left(F_{i} \dot{w}_{i}+S_{i} l_{i}\right)+\alpha K\left(\dot{w}_{2}\left(h_{2,1}^{(2)}-h_{1,2}^{(2)}\right)+\dot{w}_{3}\left(h_{3,1}^{(2)}-h_{1,3}^{(2)}\right)\right)\right. \\
&\left.+K \alpha\left(l_{1}\left(-\dot{u}_{2,2}^{(2)}-\dot{u}_{3,3}^{(2)}\right)+l_{2} \dot{u}_{2,1}^{(2)}+l_{3} \dot{u}_{3,1}^{(2)}\right)\right] d V d s \\
& \leq D\left(\int_{0}^{t} \int_{B}\left(\rho \dot{w}_{i} \dot{w}_{i} d V d s+(t-s)\left(l_{i, j}-l_{j, i}\right)\left(l_{i, j}-l_{j, i}\right)\right) d V d s\right)^{1 / 2} \\
& \times\left(\int_{0}^{t} \int_{B}\left(F_{i} F_{i}+S_{i} S_{i}+K^{2}\right) d V d s\right)^{1 / 2}
\end{aligned}
$$

where $D$ is an easily computable constant that depends on the constitutive coefficients, the initial conditions, the time $t_{1}$, and the domain.

The arithmetic-geometric mean inequality implies that

$$
\begin{aligned}
& 4 H_{\omega} \int_{0}^{t} \int_{B}(t-s)[\left(F_{i} \dot{w}_{i}+S_{i} l_{i}\right)+\alpha K\left(\dot{w}_{2}\left(h_{2,1}^{(2)}-h_{1,2}^{(2)}\right)+\dot{w}_{3}\left(h_{3,1}^{(2)}-h_{1,3}^{(2)}\right)\right) \\
&\left.+K \alpha\left(l_{1}\left(-\dot{u}_{2,2}^{(2)}-\dot{u}_{3,3}^{(2)}\right)+l_{2} \dot{u}_{2,1}^{(2)}+l_{3} \dot{u}_{3,1}^{(2)}\right)\right] d V d s \\
& \leq D^{2} H_{\omega}^{2}+4\left(\int_{0}^{t} \int_{B}\left(\rho \dot{w}_{i} \dot{w}_{i} d V d s+(t-s)\left(l_{i, j}-l_{j, i}\right)\left(l_{i, j}-l_{j, i}\right)\right) d V d s\right) \\
& \quad \times\left(\int_{0}^{t} \int_{B}\left(F_{i} F_{i}+S_{i} S_{i}+K^{2}\right) d V d s\right) .
\end{aligned}
$$

Similarly, we can obtain several constants $b_{i}$ such that

$$
\begin{gathered}
\int_{0}^{t} \int_{B} F_{i} w_{i} d V d s \leq b_{1}\left(\int_{0}^{t} \int_{B} \rho w_{i} w_{i} d V d s\right)^{1 / 2}\left(\int_{0}^{t} \int_{B} F_{i} F_{i} d V d s\right)^{1 / 2}, \\
\int_{0}^{t} \int_{B} Q_{i} l_{i} d V d s \leq\left(\int_{0}^{t} \int_{B} l_{i} l_{i} d V d s\right)^{1 / 2}\left(\int_{0}^{t} \int_{B} Q_{i} Q_{i} d V d s\right)^{1 / 2}, \\
\int_{0}^{t} \int_{B} \alpha K\left(w_{2}\left(h_{2,1}^{(2)}-h_{1,2}^{(2)}\right)+w_{3}\left(h_{3,1}^{(2)}-h_{1,3}^{(2)}\right)\right) d V d s \\
\leq b_{3}\left(\int_{0}^{t} \int_{B} \rho w_{i} w_{i} d V d s\right)^{1 / 2}\left(\int_{0}^{t} \int_{B} K^{2} d V d s\right)^{1 / 2}, \\
\int_{0}^{t} \int_{B}\left[K \alpha \left(l_{1}\left(\left(-u_{2,2}^{(2)}-u_{3,3}^{(2)}\right)-\left(-f_{3,3}-f_{2,2}\right)\right)\right.\right. \\
\left.\left.+l_{2}\left(u_{2,1}^{(2)}-f_{2,1}\right)+l_{3}\left(u_{3,1}^{(2)}-f_{3,1}\right)\right)\right] d V d s \\
\leq b_{4}\left(\int_{0}^{t} \int_{B} l_{i} l_{i} d V d s\right)^{1 / 2}\left(\int_{0}^{t} \int_{B} K^{2} d V d s\right)^{1 / 2} .
\end{gathered}
$$


Thus,

$$
\begin{aligned}
2 H_{\omega} & \int_{0}^{t} \int_{B}\left(F_{i} w_{i}+\alpha K\left(w_{2}\left(h_{2,1}^{(2)}-h_{1,2}^{(2)}\right)+w_{3}\left(h_{3,1}^{(2)}-h_{1,3}^{(2)}\right)\right)\right) d V d s \\
& \leq E H_{\omega}^{2}+H_{\omega}\left(\int_{0}^{t} \int_{B}\left(F_{i} F_{i}+K^{2}\right) d V d s\right) .
\end{aligned}
$$

In (3.13), $E$ is a constant that can be computed in terms of the constitutive coefficients, the initial conditions, the time $t_{1}$, and the domain.

In view of the estimates (2.22), (3.10), and (3.13), we can see that

$$
\begin{aligned}
& 2 H_{\omega} \int_{0}^{t} \int_{B} Q_{i} l_{i} d V d s \\
& \leq 2 H_{\omega}\left(\int_{0}^{t} \int_{B}\left(A S_{i} S_{i}+C^{*} K^{2}\right) d V d s\right)^{1 / 2}\left(\int_{0}^{t} \int_{B} Q_{i} Q_{i} d V d s\right)^{1 / 2} \\
&+2 H_{\omega}\left(B^{*} \int_{0}^{t} \int_{B} \rho \dot{w}_{i} \dot{w}_{i} d V d s\right)^{1 / 2}\left(\int_{0}^{t} \int_{B} Q_{i} Q_{i} d V d s\right)^{1 / 2}, \\
& 2 H_{\omega} \int_{0}^{t} \int_{B}\left[K \alpha \left(l_{1}\left(\left(-u_{2,2}^{(2)}-u_{3,3}^{(2)}\right)+\left(f_{3,3}+f_{2,2}\right)\right)\right.\right. \\
&\left.\left.\quad+l_{2}\left(u_{2,1}^{(2)}-f_{2,1}\right)+l_{3}\left(u_{3,1}^{(2)}\right)-f_{3,1}\right)\right] d V d s \\
& \leq N^{*} H_{\omega}\left(\int_{0}^{t} \int_{B}\left(A S_{i} S_{i}+C^{*} K^{2}\right) d V d s\right)^{1 / 2}\left(\int_{0}^{t} \int_{B} K^{2} d V d s\right)^{1 / 2} \\
& \quad+N^{*} H_{\omega}\left(B^{*} \int_{0}^{t} \int_{B} \rho \dot{w}_{i} \dot{w}_{i} d V d s\right)^{1 / 2}\left(\int_{0}^{t} \int_{B} K^{2} d V d s\right)^{1 / 2} .
\end{aligned}
$$

Again, $N^{*}$ is an easily computable positive constant. If we use the arithmeticgeometric mean inequality, we obtain

$$
\begin{aligned}
2 H_{\omega} \int_{0}^{t} \int_{B}\left(Q_{i} l_{i}+[\right. & K \alpha\left(l_{1}\left(\left(-u_{2,2}^{(2)}-u_{3,3}^{(2)}\right)+\left(f_{3,3}+f_{2,2}\right)\right)\right. \\
& \left.\left.\left.+l_{2}\left(u_{2,1}^{(2)}-f_{2,1}\right)+l_{3}\left(u_{3,1}^{(2)}-f_{3,1}\right)\right)\right]\right) d V d s \\
\leq & 4 F^{2} H_{\omega}^{2}+\left(\int_{0}^{t} \int_{B}\left(\left(C^{*}+1\right) K^{2}+A S_{i} S_{i}+Q_{i} Q_{i}\right) d V d s\right) \\
+ & \frac{B^{*} F^{2}}{2} H_{\omega}^{2}+4\left(\int_{0}^{t} \int_{B} \rho \dot{w}_{i} \dot{w}_{i} d V d s\right)\left(\int_{0}^{t} \int_{B}\left(K^{2}+Q_{i} Q_{i}\right) d V d s\right),
\end{aligned}
$$

where $F$ can be computed in terms of the constitutive coefficients, the initial conditions, the time $t_{1}$, and the domain.

From (3.5), (3.9), (3.13), and (3.15), we conclude that we can explicitly determine a constant $\xi$ satisfying (3.4).

THEOREM 3.2. Let $\left(w_{i}, l_{i}\right)$ be a solution of the problem determined by system (2.2), (2.3) with initial conditions (2.4), and boundary conditions (2.5). Then, there 
exists a positive constant $M^{*}$ such that

$$
H_{\omega}(t) \leq M^{*}\left(\int_{0}^{t_{1}} \int_{B}\left(F_{i} F_{i}+2 K^{2}+S_{i} S_{i}+Q_{i} Q_{i}\right) d V d s\right)^{1-t / t_{1}},
$$

for all $t \leq t_{1}$, where $\omega$ is given in (3.3).

Proof. If we define the function

$$
P(t)=\ln \left[H_{\omega}(t) \exp \left(\frac{1}{2} \xi t^{2}\right)\right],
$$

then

$$
\frac{d^{2} P}{d t^{2}}=H_{\omega}^{-2}\left(H_{\omega} \frac{d^{2} H_{\omega}}{d t^{2}}-\left(\frac{d H_{\omega}}{d t}\right)^{2}+\xi H_{\omega}^{2}\right)
$$

Thus, according to (3.4),

$$
\frac{d^{2} P}{d t^{2}} \geq 0
$$

Jensen's inequality gives

$$
H_{\omega}(t) \leq\left[H_{\omega}(0)\right]^{1-t / t_{1}}\left[H_{\omega}\left(t_{1}\right)\right]^{t / t_{1}} \exp \left[\frac{1}{2} \xi t\left(t_{1}-t\right)\right],
$$

for $t \in\left[0, t_{1}\right]$. The theorem is proved taking

$$
M^{*}=\max \left(1, H_{\omega}\left(t_{1}\right)\right) \exp \left[\frac{1}{8} \xi t_{1}^{2}\right] .
$$

ACKNOWLEDGment. This work is part of the project "Aspectos Matemáticos en las Teorías Termomecánicas Generalizadas” (BFM2000-0809).

\section{REFERENCES}

[1] K. A. Ames and B. Straughan, Continuous dependence results for initially prestressed thermoelastic bodies, Internat. J. Engrg. Sci. 30 (1992), no. 1, 7-13.

[2] _ Non-Standard and Improperly Posed Problems, Academic Press, California, 1997.

[3] E. Andreou and G. Dassios, Dissipation of energy for magnetoelastic waves in a conductive medium, Quart. Appl. Math. 55 (1997), no. 1, 23-39.

[4] F. Bofill and R. Quintanilla, On the dynamical and static problems in magnetoelasticity, submitted to J. Math. Anal. Appl.

[5] S. Chander, Phase velocity and energy loss in magneto-thermo-elastic waves, Internat. J. Engrg. Sci. 6 (1968), 409-424.

[6] A. C. Eringen and G. A. Maugin, Electrodynamics of Continua. I, Springer-Verlag, New York, 1990.

[7] J. E. Muñoz Rivera and R. Racke, Magneto-thermo-elasticity-large-time behavior for linear systems, Adv. Differential Equations 6 (2001), no. 3, 359-384.

[8] _ Polynomial stability in two-dimensional magneto-elasticity, IMA J. Appl. Math. 66 (2001), no. 3, 269-283. 
[9] G. Paria, Magneto-elasticity and magneto-thermo-elasticity, Adv. in Appl. Mech. 10 (1967), 73-112.

[10] G. Perla Menzala and E. Zuazua, Energy decay of magnetoelastic waves in a bounded conductive medium, Asymptot. Anal. 18 (1998), no. 3-4, 349-362.

[11] R. Quintanilla, End effects in the dynamical problem of magneto-elasticity, Arch. Mech. (Arch. Mech. Stos.) 54 (2002), no. 3, 245-256.

[12] A. J. Willson, The propagation of magneto-thermo-elastic plane waves, Proc. Cambridge Philos. Soc. 59 (1963), 483-488.

F. Bofill: Matemàtica Aplicada 2, ETSEIT, Universitat Politecnica de Catalunya (UPC), Colom 11, 08222 Terrassa, Barcelona, Spain

E-mail address: bofi11@ma2.upc.es

R. Quintanilla: Matemàtica Aplicada 2, ETSEIT, Universitat Politecnica de Catalunya (UPC), Colom 11, 08222 Terrassa, Barcelona, Spain

E-mail address: ramon@ma2.upc.es 


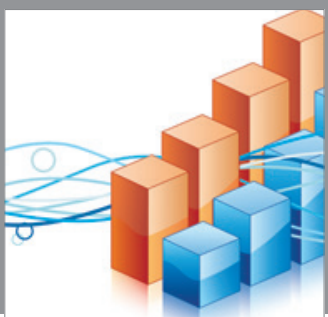

Advances in

Operations Research

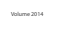

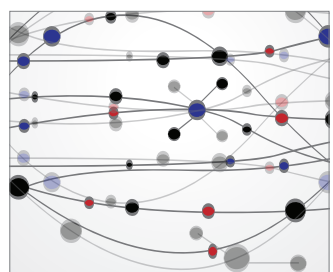

\section{The Scientific} World Journal
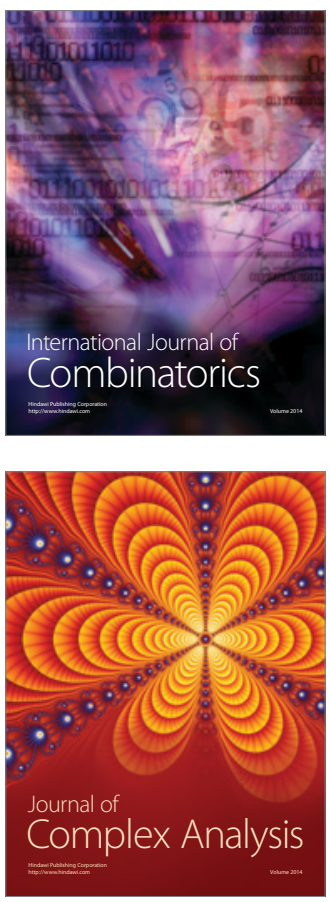

International Journal of

Mathematics and

Mathematical

Sciences
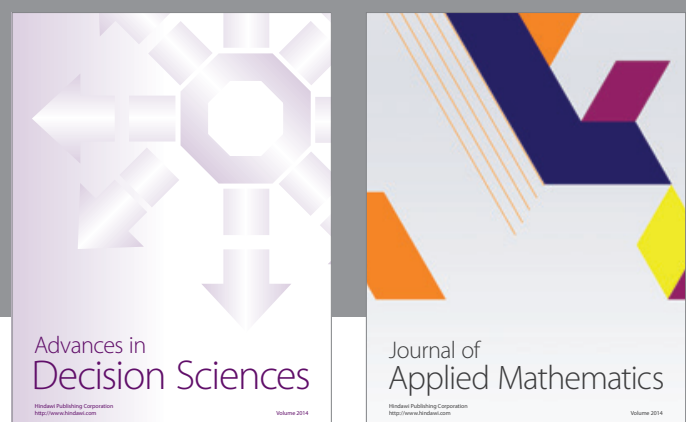

Journal of

Applied Mathematics
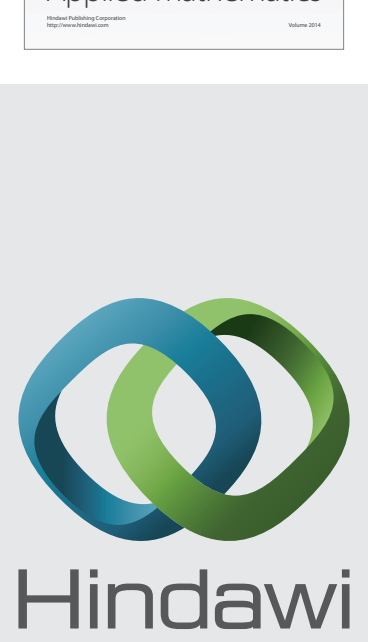

Submit your manuscripts at http://www.hindawi.com
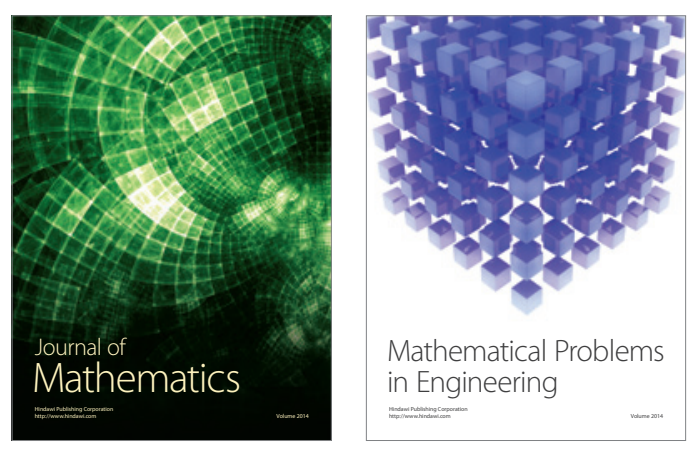

Mathematical Problems in Engineering
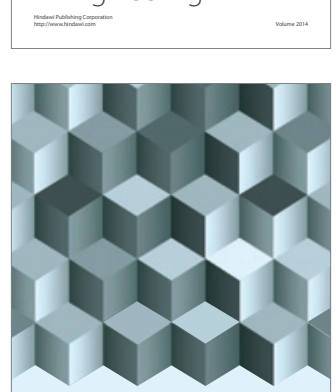

Journal of

Function Spaces
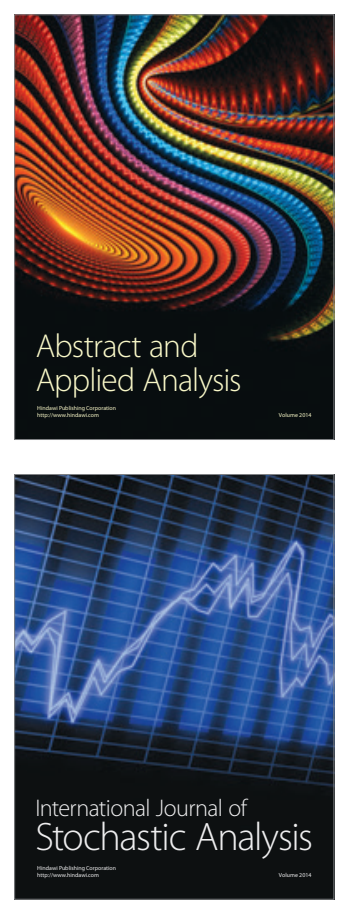

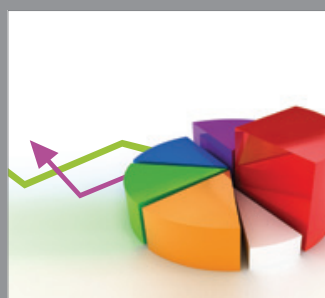

ournal of

Probability and Statistics

Promensencen
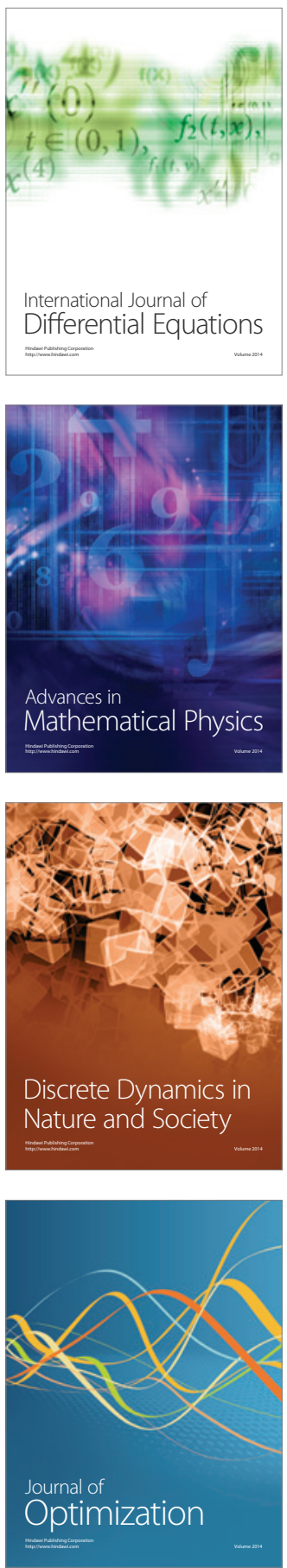